\title{
Educating Swedish telenurses to improve caller experience: Reflections on a pilot study
}

\author{
Inger K. Holmström ${ }^{1,2}$, Marta Röing ${ }^{1}$, Aniko Veg $^{1}$, Urban Rosenqvist ${ }^{1}$ \\ 1. Department of Public Health and Caring Sciences, Health Services Research, Uppsala University, Sweden. 2. School of \\ Health, Care and Social Welfare, Division of Caring Sciences, Västerås, Sweden.
}

Correspondence: Marta Röing. Address: Department of Public Health and Caring Sciences, Health Services Research, Uppsala University, Sweden. E-mail: marta.roing@pubcare.uu.se

Received: September 12, 2013

Accepted: January 9, 2014

Online Published: March 4, 2014

DOI : $10.5430 /$ cns.v2n2p61

URL: http://dx.doi.org/10.5430/cns.v2n2p61

\begin{abstract}
Telenurses employed at Swedish Healthcare Direct offer triage recommendations and self-care advice to the general public over the telephone on a wide range of health problems. Their understanding of work and ability to communicate are essential for callers' adherence to recommendations and satisfaction with the service. This paper presents and reflects upon an educational intervention for Swedish telenurses which aimed to improve caller experience. Twelve telenurses participated. Six were randomised to an intervention group, the other six to a reference group. The nurses in the intervention group individually listened to, and reflected on their authentic calls together with one of the researchers on five to six separate occasions. Callers' experiences were surveyed using a postal questionnaire sent within 48 hours of their call, both before and after the intervention. Descriptive and comparative statistics as well as content analysis were used in the analysis of the questionnaires. The most striking finding was that the educational intervention did not seem to have any effect on outcomes of the telenurses calls. Other findings revealed that a large group of callers, about $20 \%$, did not find the call useful and only $50 \%$ to $60 \%$ of the callers reported that they had learned something from the call. Callers' most frequent unfulfilled expectations centred on not being referred to a physician, and a perceived low level of competence among telenurses. The modest impact of the intervention on the services of telenursing may have been due to the participating nurses, the organisation, or the design of the intervention. Lessons have been learned that need to be taken into account in future and more large scale studies.
\end{abstract}

\section{Key words}

Caller satisfaction, Educational intervention, Reflection, Telenursing, Sweden

\section{I ntroduction}

Telephone advice nursing refers to nursing practice that focuses on "assessment, prioritization, and referral to the appropriate levels of care" for callers seeking advice or healthcare on the telephone, and includes telephone triage, or "identifying the nature and urgency of callers' needs ${ }^{[1]}$. Nurse-led telephone triage has been introduced in many Western countries, for example Holland, Australia, UK and Sweden ${ }^{[2-5]}$. Competent telenurses at well-organised call centres estimate the urgency of callers' health problems, recommend the best course of action for each individual caller, and try to steer the callers to optimal care levels ${ }^{[5-7]}$. Services provided at these call centres can in this way prevent unnecessary medical visits and result in financial savings for healthcare systems. They also have the potential to improve self-care for the public ${ }^{[5,8,9]}$. 
The national telephone helpline in Sweden, Swedish Healthcare Direct (SHD), became a new actor in the Swedish healthcare system in 2003. At present, it connects all of Sweden's 21 counties and currently employs about 1500 telenurses ${ }^{[10]}$. The number of calls has increased to 4.5 to 5 million calls yearly ${ }^{[10]}$. The main goals of SHD, one of the largest healthcare providers in the country, are to increase patients' access to healthcare services, enhance patient safety, and make the use of healthcare services more effective ${ }^{[1]}$.

In Sweden, telenurses are registered nurses, who, with the assistance of a computerized decision support system (CDSS), provide self-care advice or refer callers to an appropriate level of care ${ }^{[5]}$. This is highly skilled, knowledge-intensive work, and telenurses working in these services need to have high levels of competence in communication and also the ability to listen, as their assessments and advice are based solely on telephone communication ${ }^{[12-14]}$.

However, telenurses report anxiety about the pressures of making accurate decisions in the absence of visual cues to guide their assessments ${ }^{[15-17]}$. A recent study of telenurses' calls involved in malpractice claims revealed that the communication between telenurses and callers seemed to be nurse-driven, with few open-ended questions and a lack of exploration of callers' understanding of given advice ${ }^{[18]}$. Overall, situations in telenursing experienced as difficult by nurses are characterised by uncertainty, communication problems and insufficient resources ${ }^{[15,17,19]}$. In addition, studies have shown that telenurses encounter a range of ethical and gender-related dilemmas in their daily work ${ }^{[16]}$, and that decision aid software programmes are of limited help ${ }^{[20,21]}$, even if some studies have shown contradictory results in the latter aspect ${ }^{[5]}$.

According to Street et al. ${ }^{[22]}$, the communication behaviour of healthcare professionals might have an impact on patients' health outcomes through direct and indirect pathways ${ }^{[22]}$. A direct pathway, for example a caregiver's expression of empathy, can improve a patient's psychological well-being. More often, however, a caregiver's communication behaviour can have a positive effect on a patient's health indirectly, through the actual outcome the interaction ${ }^{[22]}$. For example, a patient's satisfaction with care or trust in the caregiver can affect patient motivation, or adherence to treatment, which may then affect health outcome ${ }^{[22]}$. These thoughts may be applied to telenurses in their interaction with callers as well. How they communicate with callers can thus greatly affect the outcome of their calls regarding the caller's adherence to recommendations, self-care, enablement and satisfaction with the call ${ }^{[23,24]}$.

There is no specific training in Sweden for telenurses, who often have different backgrounds and experiences ${ }^{[25]}$. Some telenurses have received in-house training in a so-called "dialogue process" ${ }^{[26]}$, but this model has weak scientific underpinnings. Hence, their competence and communication styles may differ significantly, which makes the quality of the call unpredictable for callers.

Recent studies have shown how educational interventions are proving to be all the more promising in continuing education in nursing ${ }^{[27-29]}$. The interventions for nurses vary, can be based on different theories, and most often explore effectiveness of workshops, lectures, or educational programs on nurses' knowledge and attitudes. This present study reports on an educational intervention for Swedish telenurses which was launced in 2004 and 2005 . The primary aim was to develop Swedish telenurses' communicative competence, and the secondary aim was to see if this could be shown in positive outcomes for the callers. A description of the intervention follows.

\section{The intervention}

\subsection{Theoretical background}

The intervention was based on theories of competence development which state that competence is not only a result of knowledge, skills and attitudes, but also a fourth aspect, namely how people understand their work ${ }^{[30]}$. According to phenomenographic theories of learning a certain phenomenon such as "work" can be understood in a limited number of ways in a group of people ${ }^{[31]}$. This has been shown repeatedly in studies from healthcare settings ${ }^{[32-34]}$. 
These theories also state that ways of understanding work will show in action ${ }^{[35]}$ and that, for example, in telenursing, different ways of performing work can be related to how the work is understood. An individual's understanding, speech and actions are inseparably intertwined within a non-dualistic world-view ${ }^{[30,35]}$. Thus, in the case of telenursing, these theories might have an impact on the outcomes of the call. If telenurses develop their understanding of work in a more comprehensive direction, this change should make them work in more competent ways and, hence, benefit callers as well ${ }^{[32]}$.

The intervention for the telenurses was educational, based on the assumptions of reflective learning as outlined by Donald Schön, who proposed reflection as a learning tool in professional education ${ }^{[36]}$. It was hypothesized that a researcher, by listening to and commenting on a telenurse's dialogues (together with the telenurse), could enhance the telenurse's reflective processes, and facilitate or alter (if necessary and possible) her understanding of the ways she worked and communicated with callers. This technique had been previously described by Holmström et al. ${ }^{[37]}$, who successfully managed to help participants reflect on video recording of their encounters with patients, and which resulted in a change of understanding of the patient-professional encounter, and thus further development of competence, for some of the participants ${ }^{[37]}$.

\subsection{Outcome of the intervention for the telenurses}

The contents of the dialogues that were discussed in the reflective sessions between the telenurses and researcher were analysed using qualitative content analysis, and have been presented in a recently published paper ${ }^{[38]}$. The analysis gave insight into how telenurses experienced demands from both the surrounding society and the organisation of telenursing, and most strikingly, revealed that the telenurses did not seem to explore background information from callers ${ }^{[38]}$.

The findings of interviews with the telenurses before and after the intervention have also been presented in a separate study ${ }^{[23]}$. A phenomenographic analysis of the transcribed interviews revealed 5 different ways the telenurses understood their work, which were hierarchical ${ }^{[23]}$. Understanding the work of telenursing as 'assessing, referring and giving advice' to the caller was common to all in the group of telenurses in the study. 'Supporting' the caller implied genuine caring for, or mothering the callers, 'strengthening' the caller implied increasing a caller's self-confidence, 'teaching' the caller involved checking to see if instructions were understood, and finally 'facilitating the caller's learning' involved tailoring an individual teaching process for each caller ${ }^{[23]}$. It was interesting to note that 3 telenurses had slightly changed their ways of understandings after the intervention ${ }^{[23]}$. However, as the following presentation of the results will show, there was a very limited change in telenursing services, as indicated by the callers.

\subsection{Aim}

The aim of this study is to present and reflect upon an educational intervention for Swedish telenurses which aimed to improve caller experience.

\section{Method}

\subsection{Design and sequence of events}

The intervention had a quasi-experimental design with pre and post measures ${ }^{[39]}$. A group of 20 telenurses working at the same call centre in Sweden were informed of the educational intervention and were asked to participate both orally and in writing. The twelve who agreed to participate were randomly divided into an intervention group and a reference group. Before the intervention, questionnaires were sent to callers who had contacted each of the 12 participating nurses. Six nurses in the intervention group were then exposed to an educational intervention (description follows). At the end of this time, callers corresponding to all 12 participating nurses were sent questionnaires again and thus were surveyed before and after the intervention. The participating telenurses were also interviewed both before and after the intervention in an effort 
to capture the ways they understood the work of telenursing, and possibly any changes in their ways of understanding after the intervention, as previously mentioned in the introduction. Please see Figure 1 for a flow chart of the study.

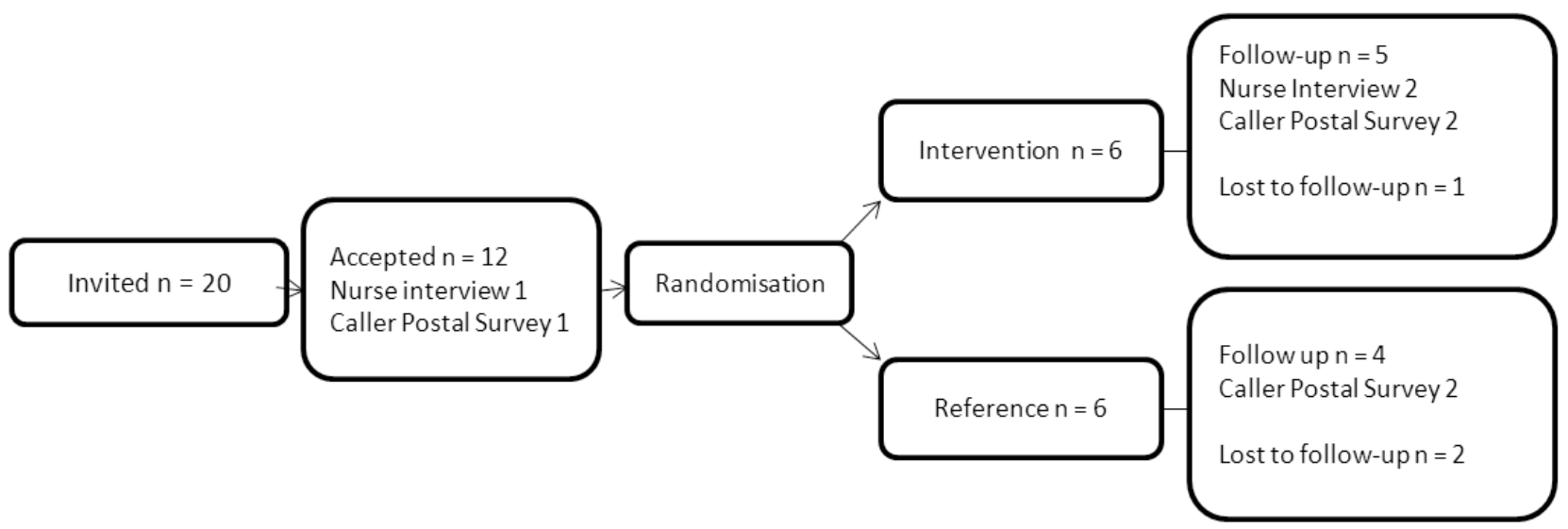

Figure 1. Flow chart of the educational intervention. 12 telenurses agreed to participate and were divided into 2 groups (intervention and reference). All telenurses were interviewed before the intervention and 5 in the interventiongroup were interviewed after the intervention. Callers were surveyed before and after the intervention for telenurses.

\subsection{Setting and sample}

The data collection was carried at one SHD site in a middle-sized town in Sweden. All 20 telenurses working at this site at the time were informed about the project and asked to participate. The twelve nurses (all female) who had consented ranged in age from 39 to 63 years, and had from four months to 35 years of experience in telenursing. These 12 nurses were then informed about the study again at a group meeting as well as in writing, and gave their informed consent. Further information was given orally in connection to the intervention sessions. The Ethics Committee at the Faculty of Medicine, Uppsala University approved the study.

All calls to this SHD site were recorded for a period of 18 months. Callers had been informed about the project in newspaper advertisements and then by a recorded message when they called SHD. They could agree or decline participation by pressing different buttons on their telephone. At the time of the study the call centre in question was open daily from 8:00 until 23:30, and handled close to 100000 calls per year. The only opportunity for telenurses to follow up on callers or get feedback about diagnosis and outcome of calls was when a patient was given an appointment to the on-duty general practitioner at the call center. Otherwise, they did not know what had subsequently happened to the patient and what the outcome of their advice had been. No other systematic review of the telenursing services took place during the study period.

\subsection{Procedure}

One of the researchers, a medical doctor with more than 15 years' experience of reflective supervision, visited the 6 telenurses in the intervention group at their workplace five to six times (for each participating nurse) during December 2004 to November 2005. On each occasion, the telenurse was given a chance to choose one day's dialogues which she wished to listen to and discuss with the researcher. After listening to a call the researcher asked the telenurse "Do you have any comments or reflections on the call we have just listened to?" He then followed up with open-ended questions (what the telenurses talked about and how they talked about it) to stimulate the telenurses' reflections on the calls and to make them curious about understandings other than their own of the topics discussed.

Example:

Researcher (R): What made you terminate the call at that particular time? 
Nurse $(\mathrm{N})$ : ...well, it was because I thought that she had grasped the message so to speak...

R: How did you come to realise that?

$\mathrm{N}$ : ...I heard it

R: How can you be sure of it? You didn't ask her...could there be ways to reassure yourself that she actually had got the message?

$\mathrm{N}$ : well, hmm...well...

Table 1. Questionnaire sent to callers before and after the educational intervention for telenurses

\begin{tabular}{|c|c|}
\hline \multicolumn{2}{|c|}{ 1. Age (year of birth) $\ldots \ldots \ldots \ldots \ldots \ldots \ldots$} \\
\hline 2. Sex & $\square$ male $\quad \square$ female \\
\hline \multicolumn{2}{|c|}{ 3. What language do you speak at home? } \\
\hline 4. Are you: & $\begin{array}{l}\text { married or living together with your partner } \\
\text { widow/widower } \\
\text { divorced } \\
\text { single }\end{array}$ \\
\hline
\end{tabular}

The last time you called Swedish Healthcare Direct:

5. What was your reason for calling?

\section{What advice did you get from the nurse?}

\section{Was this advice useful? yes \\ no}

If yes, in which way?

If no, describe what you had expected!!

8. Did you learn anything from your call? yes

no

9. Did you have contact with any other caregiver for the same reason you called Swedish Healthcare Direct?

yes

no

If yes, who did you contact?

10. Did you have any difficulties in your contact with Swedish Healthcare direct?

yes

no

If yes, what were the difficulties?

Thank you for your participation!

The researcher's intervention strategies included giving confirmation and asking the telenurses for feedback, asking for interpretations, asking reflective questions, giving suggestions, or correcting. The discussions included reflections on the nurse's understanding of the call, the measures she had taken to help the caller, the communication, the pedagogy used and the telenurse's way of encountering the caller. The educational intervention for each telenurse took a period of up to 6 months, as her sessions with the researcher were held at 4 week intervals. For the researcher, the sessions with the 
telenurses took a period of 18 months, as it was not practically possible to have ongoing sessions with more than two telenurses at a time. A total number of 121 dialogues were listened to and discussed by the 6 telenurses and the researcher. One telenurse participated in two supervisory sessions only, as she changed her place of work.

\subsection{Questionnaire to callers before and after the nurse intervention}

The questionnaire was developed by the authors for the present study. It was repeatedly discussed and revised by members of the research group, and at research seminars, and was tested for face-validity on a small group of people (6 individuals) who had called the SHD. They found it easy to understand and answer, and hence no changes were made. In the questionnaire the callers were asked about demographic factors, reason for calling, usefulness and learning experiences of the call, and whether they had experienced any difficulties related to the call or had contacted another care provider in connection to the call. They were also able to make free comments.

A total of 173 randomly selected callers per nurse were sent a postal questionnaire within 48 hours of their call. The number of callers was based on the notion that the proportions of callers who had learned anything from the call would increase from 0.2 to 0.4 using an a-coefficient of 0.5 and power of $0.98^{[40]}$. Two reminders were sent. A total of 2076 questionnaires (173 callers times 12 nurses) were sent out before the intervention and 1730 after, as two telenurses in the reference group had left the study, one changed her place of work and the other due to sick leave (173 callers times 10 nurses). A translation of the questionnaire from Swedish is presented in Table 1.

\subsection{Data analysis}

Statistical analyses of the questionnaires were performed using SPSS version 15.0 and Sphinx v.5.1.0.7 software. Descriptive statistics were used for demographic factors (sex, age, marital status, language spoken at home). All survey questions which gave the caller a choice between yes or no (usefulness of advice, learning experiences of call, contact with other caregiver, difficulties in contact with SHD) were analysed statistically. Chi-square tests were used to examine differences in callers' assessment of telenursing service before and after the educational intervention for telenurses. Content analysis ${ }^{[39]}$ was carried out on the free written comments or remarks made by the callers, and was directed by the content areas in the questionnaire. The software Atlas Ti was used in the coding process.

\section{Results - Outcome of the intervention as indicated by the callers}

The outcome of the intervention as indicated by the callers was summarised and labelled in the following categories: (1) Caller characteristics; (2) Usefulness of calling SHD; (3) Learning experiences; (4) Difficulties; (5) Other contacts; (6) Free comments regarding unfulfilled expectations. Descriptions of each category, illustrated by quotations from the free written comments, follow.

\section{1 Caller characteristics}

The recruitment and collection of the required 173 questionnaires per telenurse took between two to three months, both before and after the intervention. A total of 1155 (out of 2076) pre-intervention callers, and 987 (out of 1730) post-intervention callers returned the questionnaires after two reminders, a response rate of 55.7 and $57.0 \%$ respectively. Among the callers, about $25 \%$ were male on both occasions. Most callers ( $85 \%$ ) spoke Swedish fluently on both occasions. The most common reasons for calling in the total sample were ear, nose and throat symptoms (13.1\%), followed by request for information (9.7\%) gastrointestinal symptoms (9.1\%), common cold and cough (7.6\%) and fever 7.0\%. Characteristics of the callers are shown in Table 2 . 
Table 2. Characteristics of callers to Swedish Healthcare Direct (SHD) participating in the study before and after an educational intervention for telenurses

\begin{tabular}{lll}
\hline & Before intervention & After intervention \\
\hline Sex (f/m) & $827 / 326$ & $776 / 211$ \\
Age (mean \pm SD) & $40.7 \pm 16.3$ & $40.5 \pm 15.6$ \\
Fluent Swedish speaker & $86.6 \%$ & $87.2 \%$ \\
Civic status (total $n)$ & 1155 & 987 \\
Married, cohabiting & 802 & 727 \\
Bereaved & 30 & 27 \\
Divorced & 64 & 45 \\
Single & 236 & 167 \\
\hline
\end{tabular}

\subsection{Usefulness of calling SHD}

Before the intervention, $73.2 \%$ of callers in the reference group and $77.2 \%$ in the intervention group found the call to the telenursing service useful. After the intervention, $83.4 \%$ in the reference group, and $81.8 \%$ in the intervention group found the call useful. This change is significant in the reference group (Chi2-test, $p=0.02$ ).

Example of a useful call:

27: I had called so many places and nobody answered. I finally got an answer [from SHD] to my questions and worries about having a high fever.

Example of advice or call experienced as non-useful:

442: I wanted a better explanation about how to manage the symptoms myself. Why do I cough up blood?

\subsection{Learning experiences}

Before the intervention, $57.7 \%$ of the callers in the reference group and $53.8 \%$ in the intervention group felt they had learned something as a result of their call to SHD. After the intervention, $58.2 \%$ in the reference group and $57.1 \%$ in the intervention group felt they had learned something. There were no significant changes before and after the intervention in either the intervention or reference group. Callers' responses to each individual telenurse who participated in the intervention and reference groups as to both usefulness and learning experiences of their calls are presented in Table 3.

Example of a caller who had a positive learning experience when calling SHD:

25: I gave paracetamol since I was told that kids aged one year or less have a brain which is not yet fully developed, and hence might have episodes of cramps. I was confirmed that my thinking had been right about my son, for example that he wasn't somnolent and that it was nothing serious.

However, there were also callers who stated that the lesson they had learned was that calling SHD for health problems was of no use to them at all.

\subsection{Difficulties}

Before the intervention, $26.0 \%$ of the callers in the reference group and $22.1 \%$ in the intervention group experienced difficulties in connection to their call to the telenursing service. After the intervention, $16.0 \%$ of the callers in the reference group and $16.6 \%$ in the intervention group experienced difficulties in connection to their call. The decrease was significant in both groups (Chi2-test, $p=0.03$ resp $p=0.01$ ). 
Example of difficulties:

10: I had to wait about two hours to talk to a telenurse. During this time, I felt really bad (constant vomiting). If I hadn't been forced to wait so long I could have avoided this suffering.

Table 3. Effect of coaching a group of telenurses based on callers' reports of perceived usefulness and learning experience of their calls

\begin{tabular}{|c|c|c|c|c|c|c|c|c|c|}
\hline \multirow{2}{*}{$\begin{array}{l}\text { Intervention } \\
\text { group }\end{array}$} & \multirow{2}{*}{ Nurse } & \multicolumn{2}{|c|}{ Usefulness of the call } & \multirow{2}{*}{ CHI2 test } & \multirow{2}{*}{$p$} & \multicolumn{2}{|c|}{ Had learned something } & \multirow{2}{*}{ CHI2 test } & \multirow{2}{*}{$p$} \\
\hline & & Yes & No & & & Yes & No & & \\
\hline Before & 1 & 56 & 13 & 0.006158 & $\#$ & 38 & 20 & $2.4 \mathrm{E}-06$ & $\#$ \\
\hline After & & 74 & 8 & & & 53 & 8 & & \\
\hline Before & 2 & 85 & 16 & 0.236724 & & 53 & 27 & 0.153127 & \\
\hline After & & 75 & 15 & & & 49 & 21 & & \\
\hline Before & 3 & 81 & 33 & $4.53 \mathrm{E}-05$ & $€$ & 63 & 33 & 0.283255 & \\
\hline After & & 107 & 19 & & & 71 & 36 & & \\
\hline Before & 4 & 79 & 13 & 0.330427 & & 58 & 22 & 0.185414 & \\
\hline After & & 72 & 15 & & & 50 & 19 & & \\
\hline Before & 5 & 75 & 12 & 0.140136 & & 42 & 26 & 0.020137 & $*$ \\
\hline After & & 85 & 16 & & & 60 & 26 & & \\
\hline Before & 6 & 72 & 18 & 0.317311 & & 58 & 17 & 0.222135 & \\
\hline After & & 64 & 18 & & & 50 & 19 & & \\
\hline \multicolumn{10}{|l|}{$\begin{array}{l}\text { Reference } \\
\text { group }\end{array}$} \\
\hline Before & 7 & 67 & 14 & 0.005355 & \# & 43 & 24 & 0.006301 & \# \\
\hline After & & 94 & 14 & & & 64 & 28 & & \\
\hline Before & 8 & 76 & 30 & 0.001519 & $\#$ & 66 & 28 & 0.091685 & \\
\hline After & & 79 & 17 & & & 56 & 34 & & \\
\hline Before & 9 & 75 & 19 & 0.004395 & $\#$ & 55 & 21 & 0.375709 & \\
\hline After & & 76 & 10 & & & 49 & 20 & & \\
\hline Before & 10 & 61 & 25 & 0.001516 & \# & 57 & 17 & 0.011045 & $*$ \\
\hline After & & 89 & 20 & & & 67 & 29 & & \\
\hline
\end{tabular}

Note. Level of significance: $\left.\left.\left.{ }^{*}\right) p<0.05 ; \quad \#\right) p<0.01 ; €\right) p<0.001$

\subsection{Other contacts}

As a result of perceived difficulties during contacts with SHD, callers' contacts to other healthcare providers increased from $55.1 \%$ to $58.2 \%$ in the reference group, and from $58.3 \%$ to $60.2 \%$ in the intervention group (ns).

Example of a caller who contacted a neighbor after calling SHD:

673: I didn't get any advice about how to ease my stomach ache. She [the telenurse]could have told me that there were other medications. Instead, I called a neighbour who works at the A\&E, and she told me to take a painkiller 


\subsection{Free comments regarding unfulfilled expectations}

404 callers had made free comments about expectations from the telenursing services which had not been fulfilled. These comments were analysed and structured according to what the caller had expected. Most frequently unfulfilled expectations were as follows: the caller had expected an appointment with a physician ( $\mathrm{n}=93)$, the caller had expected effective treatment $(n=46)$, or had expected to be given effective advice $(n=62)$ or a correct assessment $(n=39)$. Others had expected a higher level of competence from the telenurses $(n=53)$ or a patient-centred consultation $(n=40)$ or to be referred $(n=17)$.

\section{Discussion and reflections}

The most striking finding in the study was that, despite the ambitious and time consuming intervention, there was a very limited change in telenursing services, according to the callers. It was hoped that the telenurses, as a result of the educational intervention, would change their ways of working, and that this would be shown in the positive outcomes of the callers. This was not the case. This raised the question of the feasibility of developing the communicative competence of the telenurses by means of the reported educational intervention. Possible answers or explanations may be found in the results from the outcome of the intervention for the telenurses ${ }^{[23]}$, as well as the callers. These are considered and reflected upon as follows.

The analysis of the callers' free written comments clearly point out that many callers expected to be referred to a physician, that they expected a higher level of competence from the nurses and that they found the suggested advice or treatment lacking in effect. This indicates that while healthcare services prioritise callers' needs, callers do not necessarily understand the prioritising function in the healthcare system. It is also possible that telenurses' assessments of symptoms were not always correct, and the suggested advice or treatment was not always effective. Here it should be noted that assessing callers' symptoms over the telephone can be a very demanding task, and also that there is no systematic follow-up as to the impact of the service or feed-back to the telenurses about how individual callers had fared after calling SHD. This may explain to some extent why there seemed to be a gap between the callers' expectations on the telenursing services and the telenurses' ways of working.

It is also evident that only $50 \%-60 \%$ of the callers reported that they had learned something from their call. Swedish nurses are well educated and should have the capacity to promote learning and health education among the public. Hence, these calls can be looked upon as pedagogic challenges for telenurses, which in turn demands a comprehensive understanding of telenursing work.

An important factor to consider is the use of guided reflection as a learning tool in the intervention. This is congruent with studies on the use of reflection and reflective practice in medical education, which state that, although this choice of approach is can be an important component of medical education, and has the potential to change a healthcare professional's understanding of their encounter with patients ${ }^{[37]}$, there is little evidence as yet that reflection actually improves patient care ${ }^{[41,42]}$. This may be due to the fact that the question of effectiveness of reflection appears to be difficult to explore ${ }^{[41]}$. While the reflective sessions may have had a slight effect on some of the telenurses 'ways of understanding' their work (as previously described), they did not have any apparent impact on their ways of working. This may have been related to the individual nurses who participated, to the organisation, or to the way the intervention was conducted. It is hard to speculate about the participating nurses. Changing one's way of working is not easy ${ }^{[43]}$. The telenurses in the study were initially motivated, and eager to be supervised and reflect on their authentic calls. However, the organisation they worked in seemed to be somewhat rigid and traditional, and calls were to be kept short. This may have hindered the nurses in trying to accept new ways of encountering the callers. The frame (the organisation of telenursing) was possibly too stable to affect, even though the intervention was carried out over a period of 18 months. An organisation will adopt changes more easily if it is large, mature and functionally differentiated ${ }^{[4]}$, which may not have been the case in this study. It is also possible that aspects of power, gender and hierarchy may have been at play in carrying 
out the intervention. The researcher who conducted the stimulated recall session with the telenurses was a senior male consultant physician. The participating female nurses might possibly have felt more at ease in reflecting upon and discussing their calls with a nurse researcher. However, the male researcher was well experienced in the method used, and had been successful with both nurses and physicians in previous interventions ${ }^{[37]}$. These factors illustrate some of the complexities of issues and problems which may have affected the outcome of the reflection sessions during the intervention.

One puzzling finding in the study was that the usefulness of the calls showed a significant increase in the reference group, and not in the intervention group. This may have been a coincidence, and may have had nothing to do with the present study. Another finding was that a rather large group of callers, about $20 \%$, reported that they did not find the call to SHD useful. These callers may have had very high expectations when making the call, such as getting an immediate appointment to a medical doctor. Anxiety, aggravating factors in the caller's previous medical history, or surrounding environment (such as long distance to medical attention) can be one reason for such a request. From a patient safety point of view, such callers identify a need for medical attendance which might be correct, and which needs to be explored by telenurses ${ }^{[18]}$.

Hence, it is not evident that the callers had over-stated their need of care. If their needs had been triaged to a lower care level, they may have been disappointed and reported this as a non-useful call. In addition, as described by Leppänen ${ }^{[4]}$, when callers express their own "diagnosis" and request medical attention, nurses do not always accept this, but instead start to question the caller, refusing to take on a role of a secretary ${ }^{[45]}$. Nurses are furthermore likely to execute power over patients who are perceived to be unpopular or difficult, as they may feel this threatens their professional competence and control ${ }^{[45]}$. Accordingly, nurses may act more like gatekeepers than carers on these occasions ${ }^{[19]}$. It has recently been shown that in a group of callers with a preconception of care level, surprisingly few callers were asked about their own opinion of being triaged to a lower level of care. This could be regarded as a nurse-driven agenda in contrast to a patient-centred agenda ${ }^{[18]}$. Callers in the present study openly requested a change in this direction in their free written comments.

In addition, nearly $60 \%$ of the callers had contacted other health care providers or relatives in connection to their call to SHD. This may have been a way of getting a second-opinion, or may have been an expression of not being reassured by advice given by the telenurses. It is important for telenurses to not only triage callers' medical needs, but also respond to emotions and concern ${ }^{[46]}$. By not acknowledging such aspects they distance themselves from callers in order to appear more professional ${ }^{[45]}$. However, emotions such as worry are highly relevant in telenursing in obtaining a complete picture of symptoms and underlying causes. If callers do not feel listened to or reassured, they may not follow given advice. This may explain why, even though three telenurses had slightly changed their ways of understanding, as previously mentioned, there was no evident impact on caller's outcomes. Thus, a change in the way of understanding does not necessarily imply a direct or an immediate change in the way of working. It should also be noted that the ways of understanding were based on how the telenurses had described their experiences of telenursing during the interviews. Even if a telenurse had changed her way of understanding work, for example from the level 'assessing, referring and giving advice' to 'supporting the caller', it is possible that the caller did not interpret or feel that he/she was being supported by the telenurse. This gives insight into the complexity of communication between human beings. As pointed out by Hughes ${ }^{[47]}$, it is essential for all nurses to develop their ability to listen. This is a key challenge for telenurses. By active listening the nurse can direct communication by paraphrasing, clarifying, providing a view of the big picture, using metaphors, acknowledging and appreciating ${ }^{[47]}$. Further research on communication in telenursing is necessary to further improve telenursing services.

A positive finding in the present study was that difficulties in the callers' contacts with SHD were perceived to have generally decreased during the intervention period. This could be an example of the Hawthorne-effect ${ }^{[39]}$. However, this may also have been due to other organisational changes which took place taking during a period of time when the SHD was rapidly expanding. When intervening in a clinical setting, there are constant on-going changes which researchers have 
no possibility to influence. This may have been the case in this study, as waiting times for callers were reduced generally during the 18-month study period.

Telenursing is still a new part of the healthcare system, and the need for further research is obvious, especially regarding communication, patient safety, medical and social effects, and the services' role in the healthcare system. There is still plenty of room for improvement. It is of outmost importance that telenurses work together with experts in medicine, communication and IT-technology to develop telenursing practice. The present intervention had a very limited impact on nurses' ways of working and on callers' experiences. Other models, theoretical approaches and intervention strategies need to be tested. A learning organisation climate is essential to develop competence and innovative ways of working ${ }^{[4]}$.

\section{Limitations}

The study did not include a representative sample of telenurses. The response rate of the caller questionnaire was above $55 \%$, which should be acceptable. Furthermore, the sample consisted of more than 2000 Swedish callers, and their characteristics were similar to Swedish callers in general (middle aged native-Swedish speaking females were in majority of callers). This study generated results which can be looked upon as preliminary. It is possible that a suitable sample size had not been recruited and that prevalence of outcome measures had been underestimated. It is also possible that face-to-face interviews with callers may have resulted in fruitful and valid data, as they could have given deeper insight into how the callers viewed their dialogues with the telenurses. This was not included in the design of the original study, as the focus of interest at the time was outcome on a group level. However, a recently published study on parents' expectations and experiences of calling SHD gives a deeper understanding of how one group of callers, parents, view their dialogues with telenurses ${ }^{[46]}$. Lessons have been learned from the present pilot study that need to be taken into account in future and more large scale studies.

\section{Conclusion}

Despite the ambitious intervention for telenurses, there seemed to be a very modest impact on the services of telenursing, as indicated by the callers. This may have been due to the participating nurses, the rigid structure of the organisation, or the design of the intervention. Power and gender aspects may also have been at play. It is also possible that the intervention should have been extended with shorter time intervals between the stimulated recall sessions. Many callers seemed to be discontent with the telenursing service. They complained about the telenurses' ways of communication, but also that they had wanted to be seen by a GP or referred to other healthcare providers, which suggests flaws in the organisation. These structural aspects should be dealt with. Many callers reported that they sought help for their symptoms from other caregivers after their calls to SHD. If this perceived need of care was due to improper judgement on the part of the telenurses is not known. A more systematic evaluation of the telenursing services seems to be needed.

\section{Acknowledgements}

We are indebted to the telenurses at the study call centre who willingly shared their experiences with us and to the callers who took their time to fill in the questionnaires. Sara Holmström gave us invaluable practical help, for which we are grateful. Grants were received from Vetenskapsrådet (The Swedish Research Council) and the Faculty of Medicine, Uppsala University, Sweden.

\section{Authors' contributions}

IKH and UR were responsible for the conception and design of the study. IH, AV and UR performed the data collection and analysis. MR and IKH drafted the manuscript. All authors (IKH, MR, AV and UR) participated in the interpretation of the findings, critical revisions for important intellectual content, and the approval of the final manuscript version. 


\section{References}

[1] American Academy of Ambulatory Care Nursing: Telehealth Nursing Practice Administration and Practice Standards. In: American Academy of Ambulatory Care Nursing. 4th edn: Pitman, NJ, USA; 2007.

[2] Derkx HP, Rethans JJ, Muijtjens AM, Maiburg BH, Winkens R, van Rooij HG, Knottnerus JA: Quality of clinical aspects of call handling at Dutch out of hours centres: cross sectional national study. Bmj. 2008; 337:a1264. PMid:18790814

[3] Keatinge D, Rawlings K: Outcomes of a nurse-led telephone triage service in Australia. Int J Nurs Pract. 2005; 11(1): 5-12. PMid: 15610339

[4] Lattimer V, George S, Thompson F, Thomas E, Mullee M, Turnbull J, Smith H, Moore M, Bond H, Glasper A: Safety and effectiveness of nurse telephone consultation in out of hours primary care: randomised controlled trial. The South Wiltshire Out of Hours Project (SWOOP) Group. Bmj 1998; 317(7165): 1054-1059. PMid: 9774295

[5] Marklund B, Strom M, Mansson J, Borgquist L, Baigi A, Fridlund B: Computer-supported telephone nurse triage: an evaluation of medical quality and costs. J Nurs Manag 2007; 15(2): 180-187. PMid: 17352701

[6] Andersson Bäck M: Conceptions, conflicts and contradictions at the introduction of a Swedish health call centre. PhD thesis. University of Gothenburg, Sweden; 2008.

[7] Belman S, Murphy J, Steiner JF, Kempe A: Consistency of triage decisions by call center nurses. Ambul Pediatr 2002, 2(5): 396-400. PMid:12241136

[8] Hagan L, Morin D, Lépine R. Evaluation of Telenursing Outcomes: Satisfaction, Self-Care Practices, and Cost Savings. Public Health Nursing. 2005; 17(4): 305-313. PMid: 10943779

[9] Peck A. Changing the face of standard nursing practice through telehealth and telenursing. Nurs Adm Q. 2005; 29(4): $339-343$. PMid: 16260998

[10] Center for eHealth in Sweden. 1177 Swedish Healthcare Direct [in Swedish] Invånartjänster. Center för eHälsa i samverkan. 1177 Sjukvårdsrådgivningen[Internet]. [cited 2013 October 23]. Available from http://www.cehis.se/invanartjanster/1177_sjukvardsradgivningen/

[11] Swedin B. Vårdråd direkt - sjukvårdsrådgivningar i samverkan (Health care advice directly - health care call centres in co-operation). Swedish Federation of County Councils: Stockholm; 2003.

[12] Farquharson B, Johnston M, Bugge C. How people present symptoms to health services: a theory-based content analysis. British Journal of General Practice 2011, April:267-273. PMid: 3063016

[13] Greenberg ME. A comprehensive model of the process of telephone nursing. J Adv Nurs 2009, 65(12):2621-2629. PMid: 19941546

[14] Wahlberg AC, Cedersund E, Wredling R. Bases for assessments made by telephone advice nurses. J Telemed Telecare 2005, 11(8): 403-407. PMid:16356314

[15] Forslund K, Kihlgren A, Kihlgren M. Operators' experiences of emergency calls. J Telemed Telecare 2004, 10(5):290-297. PMid: 15494088

[16] Holmstrom I, Hoglund AT. The faceless encounter: ethical dilemmas in telephone nursing. J Clin Nurs 2007, 16(10):1865-1871. PMid: 17880475

[17] Wahlberg AC, Cedersund E, Wredling R. Telephone nurses' experience of problems with telephone advice in Sweden. J Clin Nurs 2003; 12(1): 37-45. PMid: 12519248

[18] Ernesater A, Winblad U, Engstrom M, Holmstrom IK. Malpractice claims regarding calls to Swedish telephone advice nursing: what went wrong and why? J Telemed Telecare. 2012; 18(7): 379-383. PMid: 22923361

[19] Holmstrom I, Dall'Alba G. 'Carer and gatekeeper' - conflicting demands in nurses' experiences of telephone advisory services. Scand J Caring Sci. 2002; 16(2): 142-148. PMid: 12000667

[20] Ernesater A, Holmstrom I, Engstrom M. Telenurses' experiences of working with computerized decision support: supporting, inhibiting and quality improving. J Adv Nurs. 2009; 65(5): 1074-1083. PMid: 19399984

[21] Holmstrom I. Decision aid software programs in telenursing: not used as intended? Experiences of Swedish telenurses. Nurs Health Sci. 2007; 9(1): 23-28. PMid: 17300541

[22] Street RL, Jr, Makoul G, Arora NK, Epstein RM. How does communication heal? Pathways linking clinician-patient communication to health outcomes. Patient Educ Couns. 2009, 74(3): 295-301. PMid: 19150199

[23] Kaminsky E, Rosenqvist U, Holmstrom I. Telenurses' understanding of work: detective or educator? J Adv Nurs 2009, 65(2): 382-390. PMid: 19040692

[24] Moscato SR, Valanis B, Gullion CM, Tanner C, Shapiro SE, Izumi S. Predictors of patient satisfaction with telephone nursing services. Clin Nurs Res. 2007; 16(2): 119-137. PMid: 17452431

[25] Holmstrom I. Telenursing in Sweden. In: Telenursing. Edited by Kumar S, Snooks H. London: Springer; 2011. 
http://dx.doi.org/10.1007/978-0-85729-529-3_12

[26] Runius L. Giving advice in health care - the good dialogue. In: Telephone advice giving within the health care sector. Edited by Holmstrom I. Lund: Studentlitteratur; 2008.

[27] Gustafsson M, Borglin G. Can a theory-based educational intervention change nurses' knowledge and attitudes concerning cancer pain management? a quasi-experimental design. BMC Health Serv Res 2013, 13(1):328.PMid: 23958335

[28] Ke LS, Chiu TY, Hu WY, Lo SS. Effects of educational intervention on nurses' knowledge, attitudes, and behavioral intentions toward supplying artificial nutrition and hydration to terminal cancer patients. Support Care Cancer. 2008; 16(11): $1265-1272$. PMid: 18335258

[29] Patiraki EI, Papathanassoglou ED, Tafas C, Akarepi V, Katsaragakis SG, Kampitsi A, Lemonidou C. A randomized controlled trial of an educational intervention on Hellenic nursing staff's knowledge and attitudes on cancer pain management. Eur J Oncol Nurs 2006; 10(5): 337-352. PMid: 16246621

[30] Sandberg J. Understanding the basis for competence development. In: International Perspectives on Competence in the Workplace. Edited by Velde CR. Netherlands: Kluwer Academic Publishers; 2001: 9-25. http://dx.doi.org/10.1007/978-94-010-0742-9_2

[31] Marton F, Booth S. Learning and Awareness. Mahwah, NJ: Lawrence Erlbaum Associates; 1997.

[32] Bjorkman IK, Bernsten CB, Schmidt IK, Holmstrom I. The role of drug and therapeutics committees. Int J Health Care Qual Assur Inc Leadersh Health Serv. 2005; 18(4-5): 235-248. PMid: 16167640

[33] Larsson J, Holmstrom I, Lindberg E, Rosenqvist U. Trainee anaesthetists understand their work in different ways: implications for specialist education. Br J Anaesth. 2004; 92(3): 381-387. PMid: 14742329

[34] Larsson J, Holmstrom I, Rosenqvist U. Professional artist, good Samaritan, servant and co-ordinator: four ways of understanding the anaesthetist's work. Acta Anaesthesiol Scand. 2003; 47(7): 787-793. PMid: 12859297

[35] Giorgi A. An Example of Harmony Between Descriptive Reports and Behavior. Journal of Phenomenological Psychology. 1989; 20(1): 60-88. http://dx.doi.org/10.1163/156916289X00111

[36] Schön D. Educating the Reflective Practitioner. San Francisco: Jossey-Bass; 1987.

[37] Holmstrom I, Larsson J, Lindberg E, Rosenqvist U. Improving the diabetes-patient encounter by reflective tutoring for staff. Patient Educ Couns. 2004; 53(3): 325-332. PMid: 15186871

[38] Röing M, Rosenqvist U, Holmström I. Threats to patient safety in telenursing as revealed in Swedish telenurses' reflections on their dialogues. Scand J Caring Sci 2012:Epub ahead of print. PMid: 23289826

[39] Polit D, Beck C: Nursing research: generating and assessing evidence for nursing practice 8. ed. edn. Philadelphia: Wolters Kluwer Health/Lippincott Williams \& Wilkins; 2008.

[40] Lemeshow S, Hosmer D, Klar J, Lwanga S. Adequacy of sample size in health studies. Chichester: Wiley; 1990.

[41] Mann K, Gordon J, MacLeod A. Reflection and reflective practice in health professions education: a systematic review. Adv Health Sci Educ Theory Pract. 2009; 14(4): 595-621. PMid: 18034364

[42] Sandars J. The use of reflection in medical education: AMEE Guide No. 44. Med Teach. 2009; 31(8): 685-695. PMid: 19811204

[43] Kjeldmand D. The Doctor, the Task, and the Group. Balint groups as a means of developing new understanding in the physicianpatient relationship. Uppsala, Sweden: University of Uppsala; 2006.

[44] Greenhalgh T, Robert G, Macfarlane F, Bate P, Kyriakidou O. Diffusion of innovations in service organizations: systematic review and recommendations. Milbank Q. 2004; 82(4): 581-629. PMid: 15595944

[45] Leppänen V. Power in telephone-advice nursing. Nursing Inquiry. 2010;17(1): 15-26. PMid: 20137027

[46] Kaminsky E. 'If I didn't trust Swedish Healthcare Direct, I would never call' - views of making pediatric health calls. Clinical Nursing Studies. 2013; 1(3): 57-69. http://dx.doi.org/10.5430/cns.v1n3p57

[47] Hughes SA. Promoting self-management and patient independence. Nurs Stand. 2004; 19(10): 47-52; quiz 54, 56. PMid: 15612431 